\title{
Initial evaluation of dual-energy computed tomography as an imaging biomarker for hepatic metastases from neuroendocrine tumor of the gastrointestinal tract
}

\author{
Eddiel Cruz-Hernández ${ }^{1}$, Usman Mahmood ${ }^{2}$, Jennifer S. Golia Pernicka ${ }^{3}$, Viktoriya Paroder ${ }^{3}$, \\ Iva Petkovska ${ }^{3}$, Marc J. Gollub ${ }^{3}$, Jinru Shia ${ }^{4}$, Karuna Ganesh ${ }^{5}$, David D. B. Bates ${ }^{3} \wedge$ \\ ${ }^{1}$ Ponce Health Sciences University, Ponce, Puerto Rico; ${ }^{2}$ Department of Physics, Memorial Sloan Kettering Cancer Center, New York, NY, USA; \\ ${ }^{3}$ Department of Radiology, Memorial Sloan Kettering Cancer Center, New York, NY, USA; ${ }^{4}$ Department of Pathology, Memorial Sloan Kettering \\ Cancer Center, New York, NY, USA; ${ }^{5}$ Molecular Pharmacology Program and Department of Medicine, Memorial Sloan Kettering Cancer Center, \\ New York, NY, USA
}

Correspondence to: David D. B. Bates, MD. Department of Radiology, Memorial Sloan Kettering Cancer Center, 1275 York Ave, New York, NY 10065, USA. Email: batesd@mskcc.org.

Background: To evaluate quantitative iodine parameters from the arterial phase dual-energy computed tomography (DECT) scans as an imaging biomarker for tumor grade (TG), mitotic index (MI), and Ki67 proliferation index of hepatic metastases from neuroendocrine tumors (NETs) of the gastrointestinal (GI) tract. Imaging biomarkers have the potential to provide relevant clinical information about pathologic processes beyond lesion morphology. NETs are a group of rare, heterogeneous neoplasms classified by World Health Organization (WHO) TG, which is derived from MI and Ki-67 proliferation index. Imaging biomarkers for these pathologic features and TG may be useful.

Methods: Between January 2014 and April 2019, 73 unique patients with hepatic metastases from NET of the GI tract underwent DECT of the abdomen with an arterial phase were analyzed after exclusions. Using GSIViewer software (GE Healthcare, Madison, Wisconsin), elliptical regions of interest (ROIs) were placed over selected hepatic metastases by a fellowship trained abdominal radiologist. Quantitative iodine concentration (IC) data was extracted from the lesion ROIs, and the normalized IC (lesion IC/aorta IC) and relative IC (lesion IC/liver IC) for each liver were calculated. Spearman correlation was calculated for lesion mean IC, normalized IC, and relative IC to both Ki-67 proliferation and mitotic indices. Student's t-test was performed to compare lesion mean IC, normalized IC and relative IC between WHO TGs.

Results: There was very weak correlation between both normalized IC and relative IC for both Ki-67 proliferation and mitotic indices. A significant difference was not observed between normalized IC and relative IC to distinguish metastases from G1 and G2/3 tumors.

Conclusions: Our study finds limited potential for quantitative parameters from DECT to distinguish neuroendocrine hepatic metastases by WHO TG, as well as limited potential as an imaging biomarker for $\mathrm{Ki}-67$ proliferation and mitotic indices in this setting. Our findings of a lack of correlation between Ki-67 and quantitative iodine parameters stands in contrast to existing literature that reports positive correlations for these parameters in the rectum and stomach.

Keywords: Neuroendocrine tumor (NET); dual energy computed tomography (DECT); hepatic metastases; imaging biomarker; iodine quantification

Submitted Aug 15, 2020. Accepted for publication Dec 29, 2020.

doi: $10.21037 /$ qims-20-917

View this article at: http://dx.doi.org/10.21037/qims-20-917

$\wedge$ ORCID: 0000-0002-3105-156X. 


\section{Introduction}

Imaging biomarkers are defined characteristics that are measured from one or more medical images as indicators of normal biological processes, pathogenic processes, or responses to an exposure or intervention (1). Imaging biomarkers provide readily available, cost-effective, noninvasive tools that can enable tracking of a particular tumor repeatedly over time, can map the spatial heterogeneity within tumors, and can evaluate multiple different lesions independently within an individual (1). The implementation of effective imaging biomarkers with adequate sensitivity, specificity, and prognostic metrics is a critical unmet need for several types of tumors where the need for early detection is paramount (2).

Neuroendocrine tumors (NETs) are a heterogeneous group of malignancies with diverse biologic and clinical behaviors that vary according to the primary tumor origin, type of neuroendocrine cell, and pathologic features (3). The gastrointestinal (GI) tract (1) and lungs are the most common primary sites for NETs (4). The overall incidence of GI NETs increased from 1.00 case per 100,000 individuals in $1973-1977$ to 3.65 cases per 100,000 individuals in 2003-2007, with the highest increase occurring in rectal and small intestinal NETs (5). This increasing trend is in part due to heightened physician awareness and the widespread use of and improvement in diagnostic modalities (6). There is, however, little evidence that overall survival has improved for patients with GI NETs (5). The leading cause of death in GI NET cases is hepatic metastases, which are present in $30-80 \%$ of patients and represent the most important prognostic factor $(7,8)$.

The term "carcinoid" has been used to describe most NETs for several decades, after it was proposed by the World Health Organization (WHO) in 1980 (3). However, this generic description may be problematic because it falsely implies benignity. In the most recent 2017 WHO classification, carcinoids have been termed "welldifferentiated neuroendocrine tumors" and are graded according to mitotic count and/or Ki-67 proliferation index as G1 ( $<2$ mitoses $/ 2 \mathrm{~mm}^{2}$ or a $\mathrm{Ki}-67$ proliferation index $<3 \%)$, G2 $\left(2-20\right.$ mitoses $/ 2 \mathrm{~mm}^{2}$ or a Ki-67 proliferation index of $3-20 \%$ ), or G3 (>20 mitoses/2 $\mathrm{mm}^{2}$ or a Ki-67 proliferation index $>20 \%$ ) (6). When a discrepancy between mitotic count and $\mathrm{Ki}-67$ proliferation index is observed the higher grade is assigned, as these tumors usually display more aggressive histology and behavior than concordant lower-grade tumors (6). Increased mitotic count and Ki-67 proliferation index have been associated with an aggressive clinical behavior and poor prognosis (3).

Dual-energy computed tomography (DECT), also referred to as spectral CT, is used to acquire images at two distinct energy spectra, allowing for the differentiation and classification of tissues based on differences in photon absorption (9). DECT enables fast image acquisition, providing morphologic and functional information in a single imaging examination, therefore diminishing patient exposure to radiation $(10,11)$. The raw data derived from DECT may be mathematically manipulated to generate post-processed data sets, including iodine-extracted, virtual monochromatic (VMC), and virtual unenhanced (VUE) images (10). These processed images aid in the identification and characterization of tumors by improving enhancement of iodine in parenchymal tissue and detecting and quantifying materials, such as fat and blood $(10,12)$. Data obtained from DECT can also be used to create iodine maps, which may be used to evaluate and quantify tumor viability by improving depiction of local tumor invasion, adjacent organ involvement, and distant metastases (10).

Measurements of tumor viability, such as the Ki-67 proliferation index, are increasingly being recognized as important biomarkers for early and accurate assessment of patients' response to treatment $(10,13)$. Some preliminary studies have examined DECT as an imaging biomarker for Ki-67 proliferation index in rectal cancer and gastric cancer. In one retrospective study, the normalized iodine concentration (NIC) (14) value derived from DECT imaging of a rectal lesion was positively correlated with the expression of Ki-67 and hypoxia-inducible factor $1 \alpha$ (HIF$1 \alpha$ ), suggesting that DECT imaging could be a noninvasive method for predicting tumor cell proliferation and hypoxia in rectal cancer (13). Another study demonstrated a positive correlation between curve slope $\left(\lambda_{\mathrm{HU}}\right)$, iodine concentration (IC), and NIC values derived from DECT imaging of gastric lesions and Ki-67 expression (15). Quantitative parameters from DECT have also been demonstrated to be useful in the differentiation between hepatic NET metastasis and hepatocellular carcinomas (HCCs) by showing substantial differences in vascular tumor supply and capillary organization between both entities (8).

As prior studies have demonstrated the potential of iodine quantification from DECT as a biomarker for Ki-67 expression in primary tumors, the purpose of our study was to explore the potential of this technology as a biomarker in lesions that have metastasized to the liver. To the best of our knowledge, this is the first study for this purpose. 


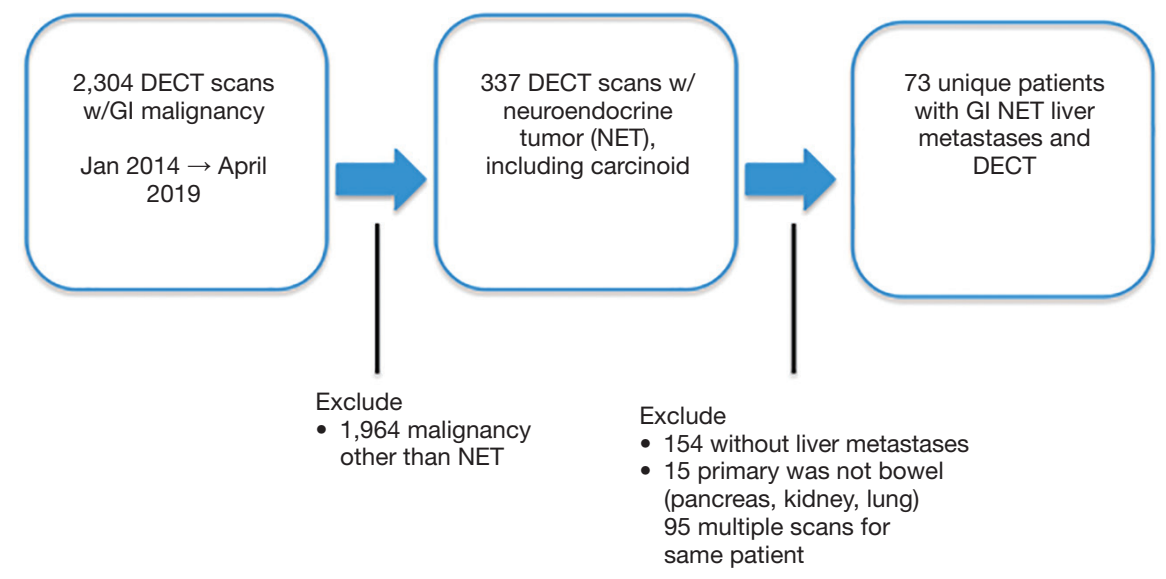

Figure 1 Flowchart is shown representing the patient selection for the study cohort. DECT, dual-energy computed tomography.

\section{Methods}

The institutional review board approved a protocol for which informed consent was waived for this retrospective project.

\section{Cobort selection}

Between January 2014 and April 2019, 2,304 DECT scans of the abdomen and pelvis were performed for patients with a malignancy of the GI tract. Of those patients, 337 had NETs, and 1,964 were excluded if there was a different primary malignancy. Of the 337 patients with DECT and NET, 154 scans were excluded for lack of hepatic metastases, 95 scans were excluded if they were repeat studies for the same patient, and 15 scans were excluded if the primary NET was not from the GI tract. This resulted in a final cohort of 73 scans for unique patients with hepatic metastases from NET of the GI tract. A summary of the patient selection process is shown in Figure 1.

\section{Image acquisition}

Images for multiphasic DECT of the liver were acquired according to standard departmental protocol on a ssDECT scanner (Discovery CT750 HD, GE Healthcare, Milwaukee, WI, U.S.). Per the protocol, patients ingested approximately $1 \mathrm{~L}$ of oral contrast $(30 \mathrm{~mL}$ iohexol in $1,000 \mathrm{~mL}$ of aqueous diluent) 1 hour prior to the exam. The arterial phase of the CT scans was selected for analysis, as NET metastases tend to be arterially enhancing. The arterial phase protocol is a acquired at $35 \mathrm{~s}$ after injection of $150 \mathrm{~mL}$ of Omnipaque 300 at a rate of $4 \mathrm{~mL} / \mathrm{s}$ with gemstone spectral imaging (GSI) enabled. Scans were acquired with rapid 80 to $140 \mathrm{kVp}$ switching, $0.7 \mathrm{~s}$ rotation time, $40 \mathrm{~mm}$ collimation, $375 \mathrm{~mA}$, helical pitch 0.984 , and a noise index of 14 .

\section{Image segmentation and quantification}

The exams were loaded in a dedicated DECT advanced workstation equipped with GSIViewer software (GE Healthcare, Madison, WI, U.S.). A fellowship trained abdominal radiologist (DDB Bates, 3 years of experience in interpreting abdominal CT post-training) reviewed the scans in GSIViewer. Iodine maps were viewed in conjunction with $70 \mathrm{keV}$ arterial phase images to select the dominant hepatic metastasis for analysis. The selected metastases were chosen, one per scan, to be the largest NET metastasis free from streak artifact that may alter quantitative assessment. An elliptical region of interest (ROI) was placed over the select hepatic metastasis for each patient on iodine maps and the IC was recorded. Subsequently, elliptical ROIs were drawn over the background normal liver parenchyma for each patient and IC values were recorded. Finally, an elliptical ROI was drawn for each patient over the abdominal aorta at the level of the liver, with care to exclude the peri-aortic tissues, and ICs were recorded.

After extracting quantitative parameters from the scans, the mean IC for each lesion was used to calculate the normalized IC and relative IC for each liver lesion using the following equations: 


$$
\begin{aligned}
& \text { Normalized iodine concentration }=\frac{\text { Lesioniodine concentration }}{\text { Aortaiodine concentration }} \\
& \text { Relativeiodine concentration }=\frac{\text { Lesion iodine concentration }}{\text { Background liver parenchymaiodine concentration }}
\end{aligned}
$$

\section{Statistical analysis}

Statistical evaluation included Spearman correlation calculated for lesion mean IC, normalized IC, and relative IC to both $\mathrm{Ki}-67$ proliferation and mitotic indices. Additionally, Student's $t$-test was performed to compare lesion mean IC, normalized IC and relative IC between WHO G1 tumors and G2/3 tumors (Figure 2).

\section{Results}

The final cohort included 73 patients $(33$ male, 40 female; mean age 63.8 years; range, 25.7-86.3). The site of the primary tumor, in descending order, was small bowel $(\mathrm{n}=57)$, colon or rectum $(n=8)$, gastrointestinal origin not otherwise specified ( $n=3)$, duodenum $(n=2)$, stomach $(n=2)$, and mesentery $(\mathrm{n}=1)$. The key results are summarized in Table 1 .

In the final cohort, 33 patients had lesions that were pathologically WHO grade 1,35 that were WHO grade 2 , and 5 that were WHO grade 3 . The mean time from initial diagnosis of NET to the time of the scan was 6.59 years (range, 0.24-23.48 years), and the median time was 5.05 years. Of the included patients, 67 were on systemic therapy, and 6 were on observation only.

The normalized IC of hepatic metastases was very weakly correlated with the mitotic index $(-0.0888)$ and $\mathrm{Ki}-$ 67 proliferation index $(0.0043)$. The relative IC of hepatic metastases was also very weakly correlated with the mitotic index (0.0702) and Ki-67 proliferation index (0.131).

The normalized IC of hepatic metastases from gastrointestinal NET was not significantly different between WHO grade 1 and grade $2 / 3$ tumors $(\mathrm{P}=0.8453$; $95 \% \mathrm{CI}$ :

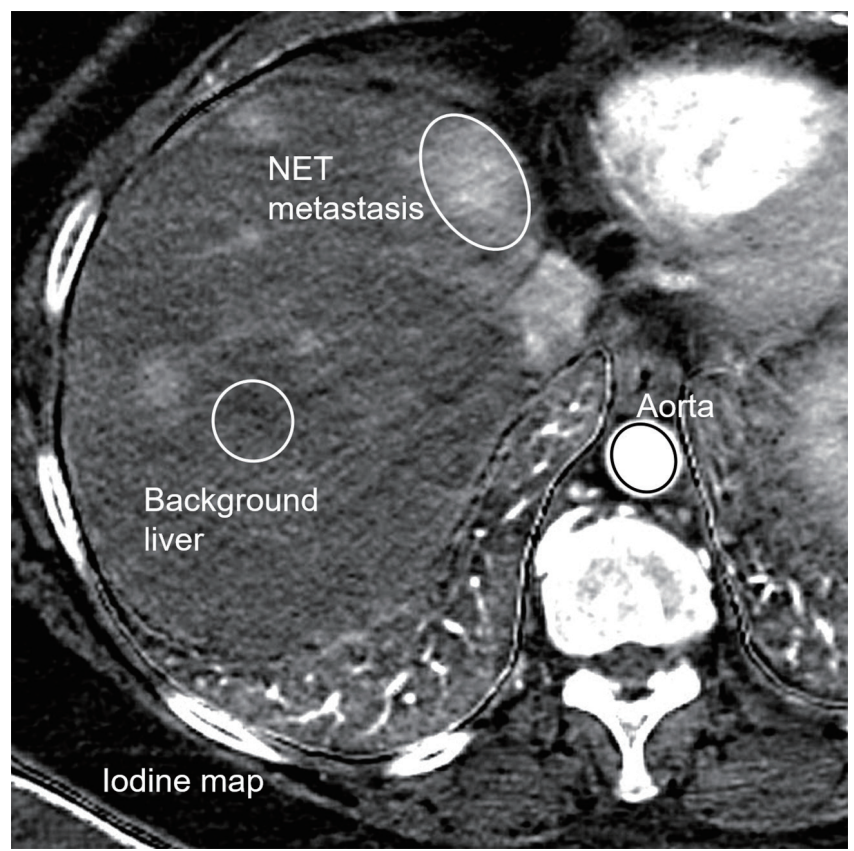

Figure 2 A 71-year-old female with grade 1 neuroendocrine tumor of the ileum and hepatic metastases. Representative image showing an arterial phase iodine map derived from DECT through the upper abdomen. Sample segmentations are shown, with an elliptical region of interest (16) over the neuroendocrine tumor metastasis, the normal background hepatic parenchyma and abdominal aorta. Extracted iodine concentrations are then used to calculate the normalized and relative iodine concentrations for analysis. DECT, dual-energy computed tomography. 
Table 1 Cohort demographics

\begin{tabular}{|c|c|}
\hline Demographics & Value \\
\hline \multicolumn{2}{|l|}{ Male } \\
\hline Female & $40(54.8)$ \\
\hline Mean age in years (range) & $63.8(25.7-86.3)$ \\
\hline Small bowel & $57(78.1)$ \\
\hline Colon/rectum & $8(11.0)$ \\
\hline GI primary NOS & $3(4.1)$ \\
\hline Duodenum & $2(2.7)$ \\
\hline \multicolumn{2}{|l|}{ Spearman correlation results } \\
\hline Normalized IC and mitotic index & -0.0888 \\
\hline Normalized IC and Ki-67 index & 0.0043 \\
\hline Relative IC and mitotic index & 0.0702 \\
\hline Relative IC and Ki-67 index & 0.131 \\
\hline \multicolumn{2}{|l|}{ Student's $t$-test results (95\% Cl; P value) } \\
\hline Normalized IC comparing WHO G1 vs. G2/3 tumors & -0.0287 to $0.0349 ; 0.8453$ \\
\hline Relative IC comparing WHO G1 vs. G2/3 tumors & -0.6933 to $0.2746 ; 0.3915$ \\
\hline
\end{tabular}

NOS, not otherwise specified; IC, iodine concentration; WHO, World Health Organization.

-0.0287 to 0.0349$)$, nor was the relative IC $(\mathrm{P}=0.3915 ; 95 \%$

CI: -0.6933 to 0.2746 ) (Figures 3,4 ).

\section{Discussion}

In our cohort, the normalized IC and relative IC of hepatic metastases from gastrointestinal NET were not significantly different for low grade (G1) and higher-grade (G2/3) tumors. In addition, the iodine quantification parameters showed only weak correlations $\mathrm{Ki}-67$ proliferation and mitotic indices.

Given the lack of significant difference between the quantitative iodine parameters of different WHO tumor grades for hepatic metastases from gastrointestinal NET, there appears to be limited potential for this tool as an imaging biomarker for this indication. It should be noted that this is specific to iodine maps created from an arterial phase CT angiogram of the abdomen, and that these results do not speak to the utility of iodine quantification for distinguishing WHO tumor grade for other phases of scanning (i.e., portal venous phase acquisition). Furthermore, the lack of correlation between relative and normalized IC of hepatic metastases on arterial phase imaging with $\mathrm{Ki}$ 67 proliferation and mitotic indices also implies limited potential as an imaging biomarker for this purpose.

Although we believe this to be the first published study assessing the role of DECT as an imaging biomarker for this purpose, the results of this study must be examined through the lens of existing literature. Regarding differentiation of gastrointestinal NET WHO tumor grade or mitotic index using DECT parameters as an imaging biomarker, we do not believe there are previously published studies on this topic. There are, however, published studies that imply some promise for iodine quantification as an imaging biomarker of Ki-67 proliferation index. Fan et al. reported positive correlation of NIC measured in primary 


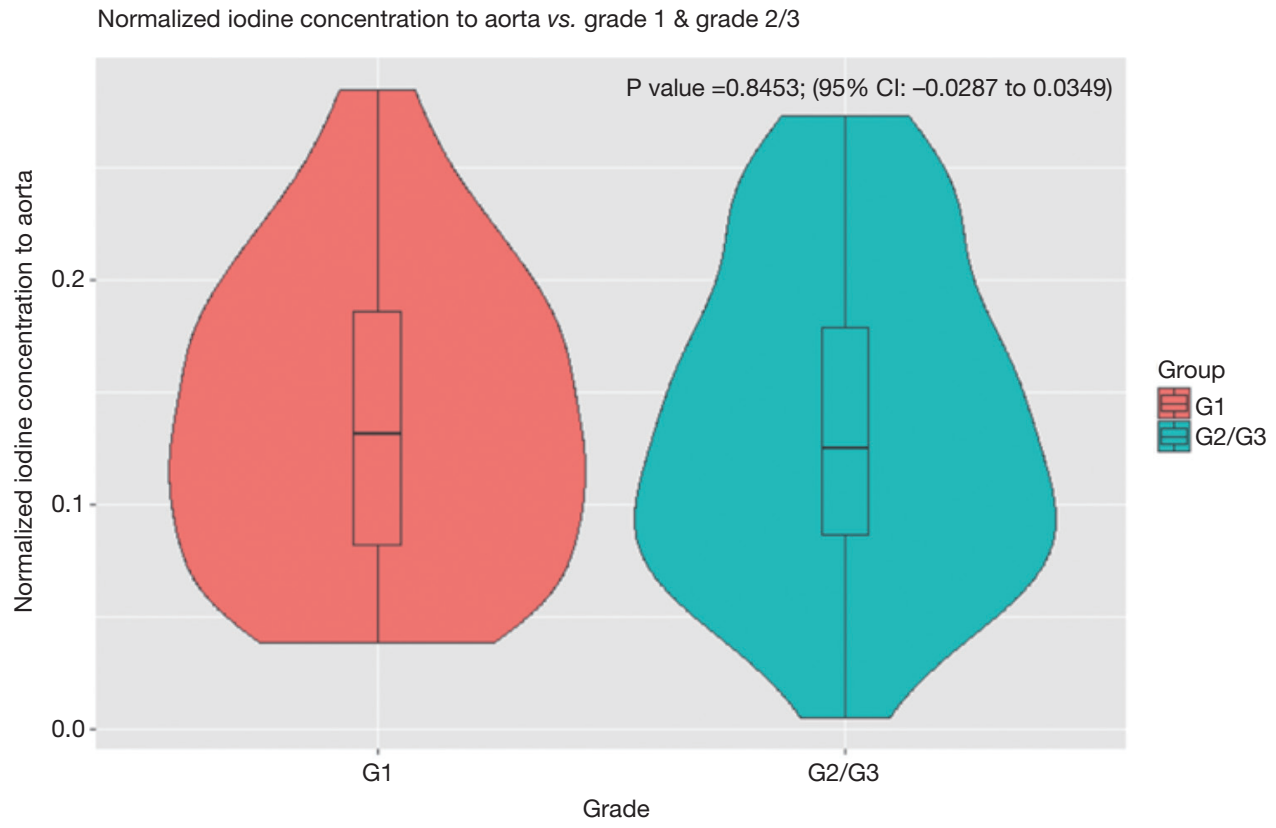

Figure 3 Violin plot showing distribution of values for normalized iodine concentration of hepatic metastases from GI NET comparing WHO G1 and G2/3. There was not a significant difference between the two groups ( $\mathrm{P}=0.8453 ; 95 \%$ CI: -0.0287 to 0.0349$)$. NET, neuroendocrine tumor; WHO, World Health Organization.

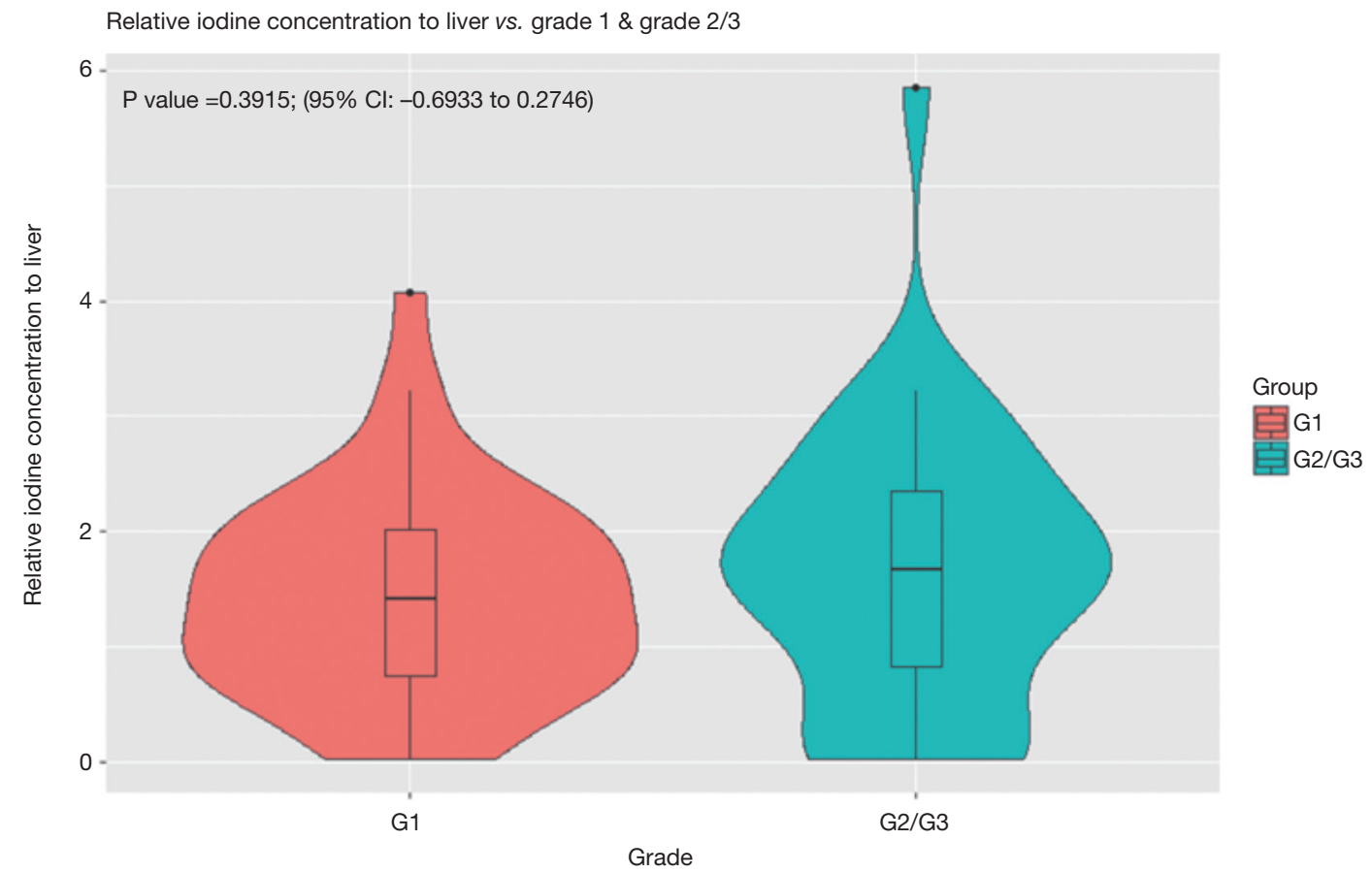

Figure 4 Violin plot showing distribution of values for relative iodine concentration of hepatic metastases from GI NET comparing WHO $\mathrm{G} 1$ and G2/3. There was not a significant difference between the two groups ( $\mathrm{P}=0.3915 ; 95 \% \mathrm{CI}$ : -0.6933 to 0.2746$)$. NET, neuroendocrine tumor; WHO, World Health Organization. 
rectal cancer with Ki-67 proliferation index (13), and Cheng et al. (15) also reported a positive correlation between IC levels and Ki-67 index in gastric cancer. Our data stands in contrast to these reports regarding the relationship between iodine quantification parameters and $\mathrm{Ki}-67$ indices, though the other studies focus on the primary tumor, whereas we have studied hepatic metastases.

The clinical implications of our study are not immediate, rather, they serve as one of several datapoints in the growing body of research around the role of DECT in oncologic imaging. The quantitative data that can be derived from routinely acquired DECT scans has tremendous potential to provide new imaging biomarkers that can help guide diagnostic imaging and therapy in the future. However, the precise role and utility of iodine quantification in DECT for oncologic imaging is still being established. It is important to recognize the limitations of DECT as a modality for finding new quantitative imaging biomarkers, and this paper that does not find a correlation for hepatic metastases from GI tract NET helps to establish one of those boundaries. How DECT becomes incorporated into the routine clinical workflow is yet to be seen.

Our study has several limitations. First, it is a relatively modest sample size with just 73 patients. In addition, scans were performed at a single institution, and may not be applicable across institutions where different CT scanners and protocols may be used. Third, this is a retrospective study, which may limit reproducibility. Lastly, nearly all of the patients were on receiving systemic treatment, and these findings may not apply to those patients who are treatment naïve.

\section{Conclusions}

In our cohort, quantitative iodine parameters derived from DECT were not able to reliably distinguish neuroendocrine hepatic metastases by WHO tumor grade. In addition, the quantitative iodine parameters did not serve as an effective imaging biomarker for Ki-67 proliferation and mitotic indices in this group. Our findings of a lack of correlation between Ki-67 and quantitative iodine parameters stands in contrast to existing literature that reports positive correlations for these parameters derived from primary lesions in the rectum and stomach. However, further studies on quantitative DECT parameters as a biomarker for hepatic metastases from NET may be warranted, as this was a modest sized cohort. This would be particularly interesting in a cohort of patients who have not received systemic therapy.

\section{Acknowledgments}

Research support was provided by GE Healthcare.

Funding: This research was funded in part through the NIH/NCI Cancer Center Support Grant P30 CA008748.

\section{Footnote}

Conflicts of Interest: All authors have completed the ICMJE uniform disclosure form (available at http://dx.doi. org/10.21037/qims-20-917). Dr. DDBB reports research support from GE Healthcare. The other authors have no conflicts of interest to declare.

Ethical Statement: The institutional review board approved a protocol for which informed consent was waived for this retrospective project.

Open Access Statement: This is an Open Access article distributed in accordance with the Creative Commons Attribution-NonCommercial-NoDerivs 4.0 International License (CC BY-NC-ND 4.0), which permits the noncommercial replication and distribution of the article with the strict proviso that no changes or edits are made and the original work is properly cited (including links to both the formal publication through the relevant DOI and the license). See: https://creativecommons.org/licenses/by-nc-nd/4.0/.

\section{References}

1. O'Connor JP, Aboagye EO, Adams JE, Aerts HJ, Barrington SF, Beer AJ, Boellaard R, Bohndiek SE, Brady M, Brown G, Buckley DL, Chenevert TL, Clarke LP, Collette S, Cook GJ, deSouza NM, Dickson JC, Dive C, Evelhoch JL, Faivre-Finn C, Gallagher FA, Gilbert FJ, Gillies RJ, Goh V, Griffiths JR, Groves AM, Halligan S, Harris AL, Hawkes DJ, Hoekstra OS, Huang EP, Hutton BF, Jackson EF, Jayson GC, Jones A, Koh DM, Lacombe D, Lambin P, Lassau N, Leach MO, Lee TY, Leen EL, Lewis JS, Liu Y, Lythgoe MF, Manoharan P, Maxwell RJ, Miles KA, Morgan B, Morris S, Ng T, Padhani AR, Parker GJ, Partridge M, Pathak AP, Peet AC, Punwani S, Reynolds AR, Robinson SP, Shankar LK, Sharma RA, Soloviev D, Stroobants S, Sullivan DC, Taylor SA, Tofts PS, Tozer GM, van Herk M, Walker-Samuel S, Wason J, Williams KJ, Workman P, Yankeelov TE, Brindle KM, McShane 
LM, Jackson A, Waterton JC. Imaging biomarker roadmap for cancer studies. Nat Rev Clin Oncol 2017;14:169-86.

2. Modlin IM, Bodei L, Kidd M. Neuroendocrine tumor biomarkers: From monoanalytes to transcripts and algorithms. Best Pract Res Clin Endocrinol Metab 2016;30:59-77.

3. Kim JY, Hong SM. Recent Updates on Neuroendocrine Tumors From the Gastrointestinal and Pancreatobiliary Tracts. Arch Pathol Lab Med 2016;140:437-48.

4. Pilipchuk NS, Borisenko GA. Effect of pyrilene and temechin on central and pulmonary hemodynamics in the treatment of hemoptysis. Vrach Delo 1988;(6):47-9.

5. Lawrence B, Gustafsson BI, Chan A, Svejda B, Kidd M, Modlin IM. The epidemiology of gastroenteropancreatic neuroendocrine tumors. Endocrinol Metab Clin North Am 2011;40:1-18, vii.

6. Chai SM, Brown IS, Kumarasinghe MP. Gastroenteropancreatic neuroendocrine neoplasms: selected pathology review and molecular updates. Histopathology 2018;72:153-67.

7. Cai W, Tan Y, Ge W, Ding K, Hu H. Pattern and risk factors for distant metastases in gastrointestinal neuroendocrine neoplasms: a population-based study. Cancer Med 2018;7:2699-709.

8. Kaltenbach B, Wichmann JL, Pfeifer S, Albrecht MH, Booz C, Lenga L, Hammerstingl R, D'Angelo T, Vogl TJ, Martin SS. Iodine quantification to distinguish hepatic neuroendocrine tumor metastasis from hepatocellular carcinoma at dual-source dual-energy liver CT. Eur J Radiol 2018;105:20-4.

9. Siegel MJ, Kaza RK, Bolus DN, Boll DT, Rofsky NM, De Cecco CN, Foley WD, Morgan DE, Schoepf UJ, Sahani DV, Shuman WP, Vrtiska TJ, Yeh BM, Berland LL. White Paper of the Society of Computed Body Tomography and Magnetic Resonance on Dual-Energy CT, Part 1: Technology and Terminology. J Comput Assist Tomogr

Cite this article as: Cruz-Hernández E, Mahmood U, Golia Pernicka JS, Paroder V, Petkovska I, Gollub MJ, Shia J, Ganesh $\mathrm{K}$, Bates DDB. Initial evaluation of dual-energy computed tomography as an imaging biomarker for hepatic metastases from neuroendocrine tumor of the gastrointestinal tract. Quant Imaging Med Surg 2021;11(5):2085-2092. doi: 10.21037/qims20-917
2016;40:841-5.

10. Agrawal MD, Pinho DF, Kulkarni NM, Hahn PF, Guimaraes AR, Sahani DV. Oncologic applications of dual-energy CT in the abdomen. Radiographics 2014;34:589-612.

11. Simons D, Kachelriess M, Schlemmer HP. Recent developments of dual-energy CT in oncology. Eur Radiol 2014;24:930-9.

12. De Cecco CN, Boll DT, Bolus DN, Foley WD, Kaza RK, Morgan DE, Rofsky NM, Sahani DV, Schoepf UJ, Shuman WP, Siegel MJ, Vrtiska TJ, Yeh BM, Berland LL. White Paper of the Society of Computed Body Tomography and Magnetic Resonance on Dual-Energy CT, Part 4: Abdominal and Pelvic Applications. J Comput Assist Tomogr 2017;41:8-14.

13. Fan S, Li X, Zheng L, Hu D, Ren X, Ye Z. Correlations between the iodine concentrations from dual energy computed tomography and molecular markers Ki-67 and HIF-1alpha in rectal cancer: A preliminary study. Eur J Radiol 2017;96:109-14.

14. Hope TA, Gollub MJ, Arya S, Bates DDB, Ganeshan D, Harisinghani M, Jhaveri KS, Kassam Z, Kim DH, Korngold E, Lalwani N, Moreno CC, Nougaret S, Paroder V, Paspulati RM, Golia Pernicka JS, Petkovska I, Pickhardt PJ, Rauch GM, Rosenthal MH, Sheedy SP, Horvat N.

Rectal cancer lexicon: consensus statement from the society of abdominal radiology rectal \& anal cancer disease-focused panel. Abdom Radiol (NY) 2019;44:3508-17.

15. Cheng SM, Ling W, Zhu J, Xu JR, Wu LM, Gong HX. Dual Energy Spectral CT Imaging in the assessment of Gastric Cancer and cell proliferation: A Preliminary Study. Sci Rep 2018;8:17619.

16. Calinescu AM, Vidal I, Grazioli S, Lacroix L, Wildhaber BE. Comment on "Beware of Too Aggressive Approach in Children With Acute Abdomen During COVID-19 Outbreak!". Ann Surg 2020;272:e244-5. 